\title{
Self-similarity of Operating Modes of Aviation Engine with the Use of Wireless Data Transmission
}

\author{
Serhii Serhiiovych Tovkach' \\ 1 National Aviation University, Automation \& Power Management Department, 1, Cosmonaut Komarov Ave., \\ 03058 Kyiv, Ukraine \\ e-mail: ss.tovkach@gmail.com
}

\begin{abstract}
The implementation of wireless data transmission technology and, in turn, increasing the efficiency of the functioning of the automatic control systems of the aviation gas turbine engines by determining their self-similarity of operating modes will ensure the mobility of control and diagnostics by the nodes of the gas turbine engine (GTE), the definition of resources and their use, as well as the reduction of the cost of their maintenance. The paper deals with the issue of the appropriate location of the wireless module operation of the electronic control system within the fractal and multifractal wavelet models of the individual components of the telecommunication IEEE 802.15.4 traffic and the transmission of information through the switch node of the packet switching, which allows conducting the research with the methods involving choice of parameters of telecommunication networks under the conditions of precisely and approximate given input data. The studies on the influence of mono and multifractality of network traffic for the characteristics of queuing on an overloaded mobile communication system server were conducted and the recommendations for IEEE 802.15.4 communication channels electronic turbo propeller fan engine control system was proposed.
\end{abstract}

Keywords: aviation engine, multifractal wavelet models, wireless, self-similarity, traffic.

\section{INTRODUCTION}

Currently, wireless information transfer networks are one of the main directions of development of the telecommunications industry. IEEE 802.15 (16) is a broadband wireless technology that complements the DSL (Digital Subscriber Line) and cable technologies as an alternative solution to the "last mile" problem at information transmission distances.

The intensive development of wireless networks poses problems with increasing their productivity, the need to develop algorithms that ensure timely and reliable transmission of information for automated control systems of gas turbine engines, which will increase the bandwidth utilization rate, shorten the response time of the base station to the requests of information transmission nodes, as well as provide different types of traffic.
In accordance with the studies by eminent scientists in the area of wireless automatic control systems design, the aviation engine was defined by J. Chen, W. Jiao, H. Wang, Ekram Hossain, V.V. Mandelbrot, J. Bercm; O.I. Shelukhina, A.A. Potapova., M.V. Ratynsky, R.E. Kahn, by international companies Securaplane technologies, Boeing, Dryden Flight Research Center NASA, Honewell according to the standards of EUROCAE $[1 \div 3]$, Radio Technical Commission for Aeronautics, Eurocontrol, ICAO, EASA and many others.

Important issues in the field of quality of service (QoS) the wireless data transmission for the aviation engine include the problems of planning and access control. In the QoS provisioning process, frame scheduling is required in order to determine which packet will be served first in a particular queue. In order to further improve the quality and efficiency of data transmission in IEEE 802.15 (16) networks, it is necessary 
to develop a new scheduling algorithm that will allow using bandwidth more efficiently for different types of traffic. Development of a new access control algorithm is also required to limit the number of connections in order to prevent the possibility of overflow and blocking lowpriority flows.

The studies on wireless technology traffic show the presence of self-similar (fractal) longterm dependent properties, which have a significant negative impact on the efficiency of data transmission. The study on the emergence of fractal processes is regulated by the IEEE 802.15.4 wireless standard, which, like IEEE 802.11 for Wi-Fi technology, defines the two lower levels of open systems interconnection (ISO-OSI) - physical (PHY) and media access control (MAC) - the lower sublevel of the data link layer OSI.

The relevance of the scientific and technical problem are follows, the purpose of which is the development of adaptive control systems of the GTE with the optimal algorithm for functional tasks of flexible circuit realization the factorized traffic of wireless data transmission.

The goal of this research was to define methods and control rules for improving the quality of service the telecommunication networks with packet switching by accounting for the multifractal properties of traffic during transmission IEEE 802.15.4 data in ACS aviation engines.

\section{WIRELESS SELF-SIMILARITY OF THE ENGINE OPERATING MODES}

In order to quantify and describe the pulsating structure of data traffic, in most cases it is enough to use statistical characteristics of the second order. The correlation function of the process of self-similarity (operating modes of the aviation engine) plays an important role in determining the large-scale invariance of the process "at a distance" due to polynomial decay [4].

In wireless technologies, the properties of stochastic self-similarity (fractality) satisfy the measurement of the trace of traffic. It is suggested that the degree of similarity is the form of traffic with the corresponding amplitude rationing. It is difficult to observe a clear structure for the measured tracks of traffic, but self-similarity allows taking into account the stochastic nature of many network devices and events that together affect network traffic.
Self-similarity is the existence of a correlation structure at a point that can be used to control traffic. For these purposes, on a large time scale, information on the correlation properties of the analyzed processes is provided, adjusting the actions of the overload management system. Since scaling mathematically leads to power relations in scalable quantities (power dependence of statistical characteristics), it can be concluded that, for example, wireless traffic manifests fractal properties when some of its estimated statistical characteristics exhibit power dependence in a wide time or frequency range.

Self-similarity and fractals are closely interconnected by the concept of long-term dependence, slowly damped dispersion, infinite moments, fractal dimensions, distributions with "heavy tails", dependence of spectral density $S(\omega)$ by law $1 / f$.

Let a time-discrete random process or time series $X(t), t \in Z$ be considered, where $X(t)$ is interpreted as the volume of traffic (measured in packets, bytes or bits), transmitted by the time $t$.

It is believed that the process $X(t)$ is stationary with a limitation of the correlation function $R\left(t_{1}, t_{2}\right)=M\left[\left(X\left(t_{1}\right)-m\right)\left(X\left(t_{2}\right)-m\right)\right]$ for the invariance with respect to the displacement that $R\left(t_{1}, t_{2}\right)=R\left(t_{1}+k, t_{2}+k\right)$ is for any one $t_{1}, t_{2}$ $\in Z$. It is assumed that the first two moments exist, finite and even for any one $t \in Z$. $M$ is the averaging operation; $m$ - the first central point; $\sigma^{2}$ - dispersion of the process $X(t)$. Under the condition of stationarity $R\left(t_{1}, t_{2}\right)=R\left(t_{1}-t_{2}, m=0\right)$ denote the correlation $R(\mathrm{k})$, the coefficient of correlation $r(k)=R(k) / R(0)=R(k) / \sigma^{2}$.

In order to formulate a large-scale invariance, the aggregated process is firstly determined $X^{(m)}$ for $X$ the level of association $m$. This process can be described as $X^{(m)}(i)=\frac{1}{m} \sum_{i=m(i-1)+1}^{m i} X(t)$. Then, it can be seen that $X(t)$ is divided into non-overlapping blocks of size $m$. Their values are averaged, and $i$ used as an index of blocks. The correlation function $X^{(m)}$ is denoted as $R^{(m)}(\mathrm{k})$. In the field of the stationary situation of the stationary processes considered, the following determinations of the second-order self-similarity are valid $[4 \div 6]$.

Definition. The random process $X(t)$ is precisely self-similar in the broadest sense with the Hurst index $H(1 / 2<H<1)$, if $R(k)=\left(\sigma^{2} / 2\right)$ $\left((k+1)^{2 H}-2 k^{2 H}+(k-1)^{2 H}\right)$ for any $k \geq 1$. The random process $X(t)$ is roughly self-similar in the broad sense, if $\lim R^{(m)}(k)=\left(\sigma^{2} / 2\right)\left((k+1)^{2 H}\right.$ $\left.2 k^{2 H}+(k-1)^{2 H}\right)$. 
Self-similarity of the 2nd order (in the exact and approximate sense) constitutes the basic structure, characteristic for modeling of network traffic.

Definition. A continuous time stochastic process $X_{i}\left(t \in R_{+}\right)$with stationary increments $Y_{i}=$ $X_{i}-X_{i-1}(i \in N)$ with the Hurst index $H(0.5 \leq H \leq$ 1.0) for any real, positive expansion coefficient $a$ is considered statistically self-similar if $X_{t}$ processes and a scalable process (with a time scale at) $a^{-H} X_{a t}$ have the same end-dimensional probability distribution density $\omega\left\{X_{1}, X_{2}, K, X_{n}\right\} \sim \omega\left\{a^{-H}\right.$ $\left.X_{a}, a^{-H} X_{2 a}, K, a^{-H} X_{n a}\right\}$ for all positive integers $n$; self-similar in the broadest sense, if the processes and $X_{a t}$ have the same statistical characteristics to the 2 nd order (average value $M\left[X_{t}\right]=M\left[X_{a t}\right] / a^{H}$, dispersion $\left.\sigma^{2}\left[X_{t}\right]=\sigma^{2}\left[X_{a t}\right] / a^{2 H}\right)$ and the correlation function $\left.R(t, \tau)=R(a t, a \tau) / a^{2 H}\right)[6]$.

Asymptotically self-similar in the broadest sense, if statistically the characteristics of the 2nd order for $X_{a t}$ and $a^{H} X_{t}$ are the same with a $\rightarrow \infty$.

Thus, the Hurst index $H$ of a self-similar series lies on the interval $(0.5 ; 1)$. When $H$ is approaching 1 row, it becomes more and more self-similar.

In addition to static similarity in scaling, selfsimilar processes have some quantitative properties. As a result, they can be determined by some equivalent features:

1. A hyperbolic fading correlation function of the $R(\mathrm{k}) \cong k^{(2 H-2)} L(t)$ form with $\mathrm{k} \rightarrow \infty$, where $L(t)$ - a slowly varying function on infinity $\left(\lim _{t \rightarrow \infty} L(t x) / L(t)\right)$ for all $x>0$. Hence, the series, formed by the successive values of the correlation function, diverges $\left(\sum_{k} R(k)=\infty\right)$, which is the definition of the long-term dependence of self-similar processes. The cumulative effect in a wide range of delays differs considerably from the effect observed in short-term dependent processes. Long-term dependence is the cause of longterm pulsations that exceed the average traffic levels, which can lead to overflow of buffers and cause loss and/or delay.

2. The dispersion of the sample mean decays slower than the reciprocal sample size. Considering the sequence $\left\{X_{i}^{(\mathrm{m})}, i=1,2, K\right\}$, obtained by averaging the initial sequence $\left\{X_{i}, i=1,2\right.$, $K\}$ for non-intersecting successive blocks of size $m$, the variance of self-similar processes slowly decreases by law $\sigma^{2}\left(X_{i}^{(m)}\right) \infty m^{(2 H-2)}$, while $m \rightarrow \infty$, for stationary random processes, the variance $\sigma^{2}\left(\left\{X_{i}^{(m)}, i=1,2, K\right\}\right)=\sigma^{2} m^{-1}$ decreases inversely proportional to the sample size. Hence, the average value and the variance will converge very slowly, especially when $H$ $\rightarrow 1$, it is reflected in all measures of self-similar processes.

3. If self-similar processes can be considered in the frequency domain, then the phenomenon of long-term dependence leads to a power-law nature of the spectral density in the vicinity of zero. In fact (in the case of weak regularity conditions imposed on a slowly changing function $\left.L_{1}\right), X$ is long-term dependent, if $S(\omega) \sim$ $\omega^{-\gamma} L_{2}(\omega)$, where $\omega \rightarrow \infty$, where $0<\gamma<1 ; L_{2}$ is a function that slowly changes at zero; $S(\omega)$ $=\sum{ }_{k} R(k) e^{i k \omega}-$ spectral density. Accordingly, from the point of view of spectral analysis, the long-term dependence explains that $S(0)=\Sigma$ ${ }_{k} R(k)=\infty$, that is, the spectral plane directs $k \rightarrow \infty$ when the frequency $\omega$ approaches 0 (a similar phenomenon is called $1 / f$-noise).

Consider the definition of the relationship of self-similarity in the broad sense and the correlation coefficient $r(k)$. For values $0<H<1$ and $H$ $\neq 1 / 2$ the correlation coefficient are determined $r(k) \sim H(2 H-1) k^{2 H-2}$ with $k \rightarrow \infty$. In particular, if $1 / 2<H<1, r(k)$ it behaves as $c k^{\beta}$ if $0<\beta<$ 0 where $c>0$ is the constant; $\beta=2-2 H$, then $\sum_{\mathrm{k}=-\infty}^{\infty} r(k)=\infty$.

Thus, the correlation coefficient is slowly (hyperbolically) fading, which is the main reason for the incompatibility. When the $r(k)$ fading is hyperbolic, the corresponding stationary process $X(t)$ is long-term dependent. In contrast, the process $X_{t}$ is short-term dependent if the normalized correlation function is summed $\left(\sum_{\mathrm{k}=-\infty}^{\infty} r(k)=\right.$ const $<\infty$ ). An equivalent definition can be given in the frequency region where it is necessary that the spectral density of the process, described by the expression $S(\omega)=(2 \pi)^{-1} \sum_{\omega=-\infty}^{\infty} r(\tau) e^{i k \omega}$, satisfies the condition $S(\omega) \sim c|\omega|^{-\alpha}, \omega \rightarrow \infty$ (here $c>$ 0 - the constant; $0<\alpha=2 H-1<1)$. Therefore, the spectral density $S(\omega)$ at $\omega$ approaching zero requires more low-frequency components.

Lets define several cases of the influence of different values $H$ on $r(k)$ :

- if $H=1 / 2$, then $r(k)=0$ and $X_{t}$ - an a priori short-term dependent process due to its complete lack of correlation;

- if $0<H<1 / 2$, then $\sum_{\mathrm{k}=-\infty}^{\infty} r(\tau)=0$, that is an artificial situation that rarely occurs in real applications;

- if $H=1$ there is a degenerate situation $r(k)$ $=1$ for any $k \geq 1$. 
For a self-similar process, the dispersion of the sample mean decreases slower than the magnitude of the inverse sample size $\sigma^{2}\left[X_{t}^{(m)}\right] \sim m^{-\beta}$ for a sufficiently large .

The property of a slowly damped dispersion can be detected by plotting log - log the function $\sigma^{2} \mid X_{t}^{(m)}$ from $m$ (the graph of variance dispersion). A straight line with a negative slope (less than 1 (in the wide range $m$ ) indicates a slowly damped dispersion).

\section{HURST INDEX FOR SELF-SIMILARITY THE ENGINE MODES}

Checking for self-similarity and estimating the Hurst index $H$ is a complicated task. In real terms, it always operates with end-to-end data sets, so it is impossible to check if there is a selfsimilar trace of traffic by definition. Consequently, it is necessary to investigate various properties of self-similarity in real measured traffic.

Nowadays, there are several methods for estimating self-similarity in time series: the graph of variance variation; $R / S$ - analysis; periodograms; and an estimate $H$ based on the use of wavelets.

Chart of dispersion change. The graph of variance variation is based on the following asymptotic property of the long-term process dependence $[6 \div 8]$,

$$
\operatorname{Var}\left[X^{(m)}\right]=m 2^{H-2} \operatorname{Var}[X], m \rightarrow \infty
$$

where, $X^{(m)}$ denotes a - $m$-aggregate process for $X, X_{k}^{(m)}=1 / m \sum_{t=(k-1) m}^{k m} X_{t}, m$ - the level of aggregation, $m=1,2, \ldots$.

In practice, for a $X$ given size series $n$, the maximum value $m$ is chosen such that $n / m$ is still quite large and as a result of the logarithmic application increase from $m$ to 1 to this value. For these successive values $m$, the logarithm of the sample dispersion $X^{(m)}$ is indicated by the logarithm of $m$. If $X$ is a long-term dependence, then the dispersion change schedule should be a straight line with a slope $2 \mathrm{H}-2$. Since, $0.5<H<1$ then, the asymptotic inclination of the dispersion change graph will be between -1 and 0 . The graph of the variation of the dispersion with the slope -1 will indicate that the series is not a long-term dependence and has a finite dispersion.

The Hurst index can be calculated by approximating the smallest squares to the graph points in a wide range of changes $m$.
$\mathbf{R} / \mathbf{S}$ analysis. Consider a range $X$ of sizes $n$ with selective mean $\bar{X}_{n}$ and selective dispersion $S^{2}(n)=(1 / n) \sum_{i=1}^{n}\left(X_{i}-\bar{X}_{n}\right)^{2} . \mathrm{R} / \mathrm{S}$ statistics of the normalized scale [9] for is determined on the basis of the ratio:

$$
\frac{\mathrm{R}(\mathrm{n})}{\mathrm{S}(\mathrm{n})}=\frac{\max \left\{W_{i}: i=1,2, \ldots, n\right\}-\min \left\{W_{i}: i=1,2, \ldots, n\right\}}{S(n)}(2)
$$

where $W_{i}=\sum_{k=1}^{i}\left(X_{k}-\bar{X}_{n}\right)$. It can proven that for any stationary process with a long-term relationship to the indicator $H$, the $R / S$ ratio has the following property for large $n$ :

$$
E\left[\frac{R(n)}{S(n)}\right] \sim\left(\frac{n}{2}\right)^{H}
$$

which is known as the Hurst phenomenon [9].

Given the empirical number of lengths $n\left(X_{j}: j=1,2, \ldots, n\right)$, let divide the series into $K$ blocks of size $[n / K]$. Then for each $\mathrm{d}:=[n / K]$ calculate the ratio $R\left(t_{i}, d\right) / S\left(t_{i}, d\right)$, which $t_{i}$ indicates the starting point of the data block $d$, ie $t_{i}=[n / K](i-1)+1, i=1,2, \ldots, d$. As a result, can be obtain $K$ estimates for $R / S(d)$ each value $d$. Choosing logarithmically spaced values for $(d<n)$ and putting the graph on the value $\log \left[R / S\left(t_{i}, d\right)\right]$ from $\log d$ the resulting $R / S$ graph was obtained.

Next, using the method of least squares, it is necessary to approximate the $R / S$ graph. The slope of the regression line will give an estimate of the Hurst index for long-term dependence. The smallest values $d$ should not be taken into account for this test, since in these points the short-term dependence of the series prevails; the upper values of the graph are not used, since only a few values in this area can make the estimate unstable. In practice, values $d$ in the middle of the $R / S$ graph are used to evaluate $H$.

Periodograms method. This method is used to identify the manifestation of long-range dependence in the frequency domain. For a discrete series $X=\left\{X_{1}, X_{2}, \ldots, X_{n}\right\}$ of periodograms, that is, the selective spectral power density, estimated using the Fourier transform, it is defined as:

$$
I(\lambda)=\frac{1}{2 \pi n}\left|\sum_{k=1}^{n}\left(X_{k}-\bar{X}\right) e^{i k \lambda}\right|^{2}
$$

where $\lambda$ is the frequency, $\lambda \in[0 ; \pi)$.

The basic idea of periodogram analysis is to display periodograms in a double logarithmic scale and to evaluate $H$ the slope of the regres- 
sion line, selected to the chart at low frequencies. In practice, the graph of periodograms is a graph $\left\{\log \lambda_{j}, \log I\left(\lambda_{j}\right)\right\}, j=1,2, \ldots, M$ where $\lambda_{j}=2 \pi j / n$ and $M$ is always chosen equal to $n / 4, n / 8, n / 16$, or $n / 32$ and so on depending on from how large $n$ is. According to (4) for processes of long-term dependence, the graph should be a straight line with a slope $-\gamma=1-2 H$

Wavelet estimation. The wavelet analysis of long-term traffic traffic was introduced in [9] and found that the assessment is highly unbounded and very stable in the presence of deterministic trends.

The following is a brief description of the wavelet evaluation.

Discrete wavelet transform (DWT) is a discrete series $\left\{X_{1}, X_{2}, \ldots, X_{n}\right\}$ using a combination of scalable and delayed variants of the parent wavelet function $\psi$. At scale $j$, the wavelet coefficients $d_{X}(j, k)$ are determined as follows:

$d_{x}(j, k)=2^{j / 2} \sum_{i=1}^{n} X_{i} \psi\left(2^{-j} n-k\right) j=1,2, \ldots ; k=1,2, \ldots, 2^{-j} n(5)$

Let $X$ be a stationary second order process. Then its wavelet coefficients $d_{X}(j, k)$ are satisfied:

$$
\mathrm{E}\left[\mathrm{d}_{\mathrm{x}}(j, k)^{2}\right]=\int f(\lambda) 2^{j}\left|\Psi\left(2^{j} \lambda\right)\right|^{2} d v
$$

where, $f(\lambda)$ and $\Psi(\lambda)$ - the power spectrum for and the Fourier transform for the wavelet function $\psi$, respectively. On the basis (4) can be obtained

$$
E\left[d_{x}(j, k)^{2}\right\rfloor \sim 2^{j(2 H-1)} c_{f} C(H, \psi)
$$

where $C(H, \psi)=\int|\lambda|^{-(2 H-2)}|\Psi(\lambda)|^{2} d \lambda$ - constant, which depends on $H$ and $\psi$.

If the length $X$ is equal to $n$ then the available number of wavelet coefficients in octave $j$ is $n_{j}, n_{j}$ $=2^{-j} n$ then

$$
\mu_{j}=E\left[d_{x}(j, k)^{2}\right] \approx \frac{1}{n_{j}} \sum_{k=1}^{n_{j}}\left|d_{x}(j, k)\right|^{2}
$$

The formula (7) provides a possible way to evaluate the Hurst index of long-term dependence processes:

$\log _{2} \mu_{j} \approx \log _{2}\left(\frac{1}{n_{j}} \sum_{k=1}^{n_{j}}\left|d_{x}(j, k)\right|^{2}\right) \sim(2 H-1) j+c(9)$

where, $c=\log _{2}\left(c_{j} C(H, \psi)\right)$ is a constant. This means that if $X$ is a long-term relationship with the Hurst index $H$, then the graph $\log _{2}\left(\mu_{j}\right)$ from $j$ is called the logarithmic diagram (LD) should have a linear inclination $2 H-1$.

\section{FRACTAL PROCESSES TRAFFIC FOR ELECTRONIC CONTROL SYSTEM IN AVIATION ENGINE}

The main distinguishing feature of self-similar (fractal) processes is that they cover a wide range of time scales. In the literature on teletraffic, the concept of burstiness is often used in this context. Mathematical models, which tend to cover and describe self-similar, fractal or pulsating phenomena in an economical way, use stochastic self-similar processes and duly chosen dynamic systems $[8,9]$. The general characteristic of these models is that their spatial-temporal dynamics are in some way controlled by the functions of power distribution and hyperbolically dampening correlation function. Traditional approaches to modeling fractal phenomena are based on a highly parametric multilevel hierarchy of traditional models, which, in turn, are characterized by distributions and correlation functions that fade exponentially.

The pulsating or fractal phenomena were observed in many branches of science and technology: hydrology, economics, biophysics, and others. The models that take these phenomena into account are relatively new to the theory of telephony and reflect the contemporary contribution to the already existing large class of alternative patterns of traffic description in packet switched networks. Although the use of fractal models was substantiated by many dimensions, their practical implementation and analysis were ignored, mainly due to the fact that they were viewed as the models that are almost non-analytical. A successful use of fractal models in the theory of teletraffic will depend not only on how well they describe real network traffic, but also on how great their use for network analysis and management is.

Traffic simulation was historically derived from traditional telephony and was almost exclusively based on Poisson assumptions regarding the structure of traffic revenues and exponential assumptions regarding the content maintenance requirements. However, with the appearance of modern high-speed packet switched networks (cells), there was a problem of combining new and existing transmission technologies, and switching with extremely heterogeneous mix of services and applications. As a result, packet traffic has become more complex or throttling than voice traffic, simply because it covers very different time scales (from microseconds to minutes). 
From a practical point of view, stochastic models of traffic flows are widely used for forecasting network traffic and analyzing the performance of networks, since they are able to predict the throughput of communication networks with an acceptable degree of accuracy. Additionally, confidence to traffic models is greatly increased if the model, in addition to approximating its statistical characteristics, covers the visual properties of experimentally observed traffic.

In traditional telephony, classical traffic patterns work great when designing and analyzing system performance as a whole.

However, with the appearance of modern high-speed communication networks (for example, B-ISDN in ATM) there is a heterogeneous mix of traffic. The pulsating structure inherent in some widely used B-ISDN-services (mainly compressed video and file transfers) determines the failure of traditional models for short-term and long-term dependencies.

Multifractal property. Unlike self-similar processes, the multi-scale or multifractal processes provide a more flexible law of scale behavior. The class of multifractal processes includes all processes with the property of scaling, including self-similar, monosensitive and large-scale processes. The most obvious examples of large-scale processes are multiplicative cascades.

Definition 1. $[9,10]$ The stochastic process $X(t)$ is called multifractal, if it has stationary increments and satisfies:

$$
\left.M \|\left. X(t)\right|^{q}\right\rfloor=c(q) t^{\tau(q)+1}
$$

for some positive $q=Q[0,1] \subset Q$, where $\tau(q)$ is called the mass index (scale function) and moment coefficient $c(q)$ which is not depend on $t$.

The obvious consequence of this definition is that $\tau(q)$ is a convex function. If $\tau(q)$ linearly dependent on $q$, then the process is called singlescale or monofractal, otherwise it is multifractal.

QoS for traffic maintenance. The analysis of the state the technical equipment shows that in the near future the tendency to construct integrated, multiservice networks will continue. As the current status of the Internet network shows, processing of all "equal rights" traffic under conditions of limited bandwidth leads to serious problems. Hence, forwarding the most important data may be temporarily blocked by transferring a large file. Therefore, when creating a TQ with combined functions it is necessary to guarantee the necessary level of service for each application. Otherwise, users will be forced to abandon the multiservice network in favor of the highly specialized one.

Most problems arise while trying to "collect" a set of highly specialized networks into one multiservice network. It is even harder to obtain such a network that could solve all problems. Network functions may change during the operation due to the emergence of new services. The changes in network-oriented applications impose new requirements on TQ.

There was a need to guarantee the response time, network bandwidth, packet delay, jitter, percentage of lost packets and other network settings. This concept was developed and named the quality of service QoS (Quality of Service).

The quality of service is a connecting link of the user and system. Changing QoS will always be noticed by users, especially as it relates to realtime, such as video or speech transmissions.

The concept of quality of service is regulated and implies specific performance indicators of the system.

The quality of service is evaluated on the receiving side. For a simplified description of the notion of service quality, three parameters which should characterize QoS are distinguished. The following parameters reveal the notion of service quality.

Time delay for packet transfer is the maximum packet transfer time from one node to another. This parameter depends on the latency of the transmission and the time the packets are located in the queue.

The delay variation reflects the difference between the maximum and minimum packet transfer time between nodes. This value depends on the number of virtual connections multiplexed into one physical channel. In addition, it is affected by the change in packet delay time in the switches queues.

The percentage of lost packets depends on the quality of a particular physical channel and the algorithm put in the switch to eliminate overload.

It can be conditionally split traffic into three categories that differ in one from one requirement to delay, jitter and a percentage of loss during transmission.

Real time traffic. It includes the audio and video information critical to transmission delays. The acceptable values of delays usually do not exceed $0.1 \mathrm{~s}$ (this includes time for processing packets by the terminal station). In addition, the delay should have small fluctuations (with them the effect of jitter). While compressing informa- 
tion, the traffic of this category becomes very sensitive to transmission errors, and due to stringent requirements for delays in the transmission of streams in real-time, the occurring errors cannot be corrected by retransmission.

Transaction Traffic. For this type of traffic, the delay should not exceed $1 \mathrm{~s}$. Otherwise, users will be forced to interrupt their work and wait for response to their messages, because they can only continue to send their data after receiving a response. Such a scheme of information exchange reduces productivity, and the spread in the values of delays can lead to a sense of discomfort from users. In some cases, exceeding the allowable delay time leads to a crash of the work session.

Data Traffic. Delays in the transmission of traffic in this category can have virtually any meaning and reach even a few seconds. For such traffic, the bandwidth is more important than the delay time: an increase in network bandwidth entails a reduction in transmission time. The attachments that transmit large volumes of data are designed to basically capture the entire available bandwidth of the network. The only exceptions are streaming video applications. For them, both bandwidth and minimization of delay time are important.

Within each category, traffic can be allocated by priority. High-priority traffic has advantages in processing because of its importance for use for specific purposes. The introduction of priorities is inevitable in the absence of network resources. Priorities can be used to highlight groups, applications, and individual users in groups.

The transmission of audio and video information is sensitive to delays or jitter changes. For example, exceeding the permissible threshold of a jitter can lead to tangible distortion of images, the need to duplicate video frames, etc. Language transmissions are also sensitive to jitter, since it is difficult for people to perceive unexpected pauses in the language of the subscriber.

In addition, since audio and video streams flow through various devices that handle traffic based on a jitter effect based on different algorithms, the synchronization between image and voice may be lost quickly. The effect of jitter can be decreased by applying buffer memory on the receiving side. However, it should be remembered that the volume of the buffer can reach significant size, which leads to a rise in price of hardware, as well as the reverse effect, i.e. an increase in delay due to overhead when processing information in a large buffer.
Influence of mono and multifractal traffic on QoS. As noted above, the self-similarity of traffic, as a rule, has a significant impact on the performance of the system and the quality of service. There are currently no systematized studies devoted to the study of the effect of self-availability on the quality of service in wireless communication systems.

The research in this area, especially when considering multifractal telecommunication traffic, is not presented in widely available publications.

The main task of the research carried out in the study on the mono and multifractal properties of wireless data traffic, and assessment of their impact on the characteristics of the quality of service to ensure given by QoS. For solving this problem, algorithms and software for numerical estimation of influence of multifractal traffic on parameters of quality of service must be developed.

\section{WIRELESS TECHNICAL IMPLEMENTATION FOR TURBOPROPFAN AVIATION ENGINE}

For the technical implementation and improvement of the quality of service of wireless communication in the SAC GTE with packet switching due to the account of multifractal properties of traffic, it is expedient to determine the self-similarity of the operating modes of the engine is the use of "distributed networks" (scatternet), which include piconets with Adaptive Frequency Hopping (AFH) technology. AFH avoids collisions: during interchanging information, spectrum signals are used by frequency hopping (Frequency Hop Spread Spectrum) over a multifractal process.

Permanent frequency interchanges allow the radio interface to broadcast information over a range of frequencies and significantly reduce the impact of interference from the devices that operate in the same range. If this channel is noisy, the system will switch to another one, and this will take place until the channel is determined to be free from interference.

In Figure 1 the frequency-time plane is shown, which illustrates the simultaneous operation of modules of wireless data transmission.

By using FHSS, data is split into small packets and transmitted at different frequencies. The first packet with the speed of frequency "jumps" per second is sent at one frequency, followed by packages - on the other. The scheme of transitions is determined by a special algorithm; the 


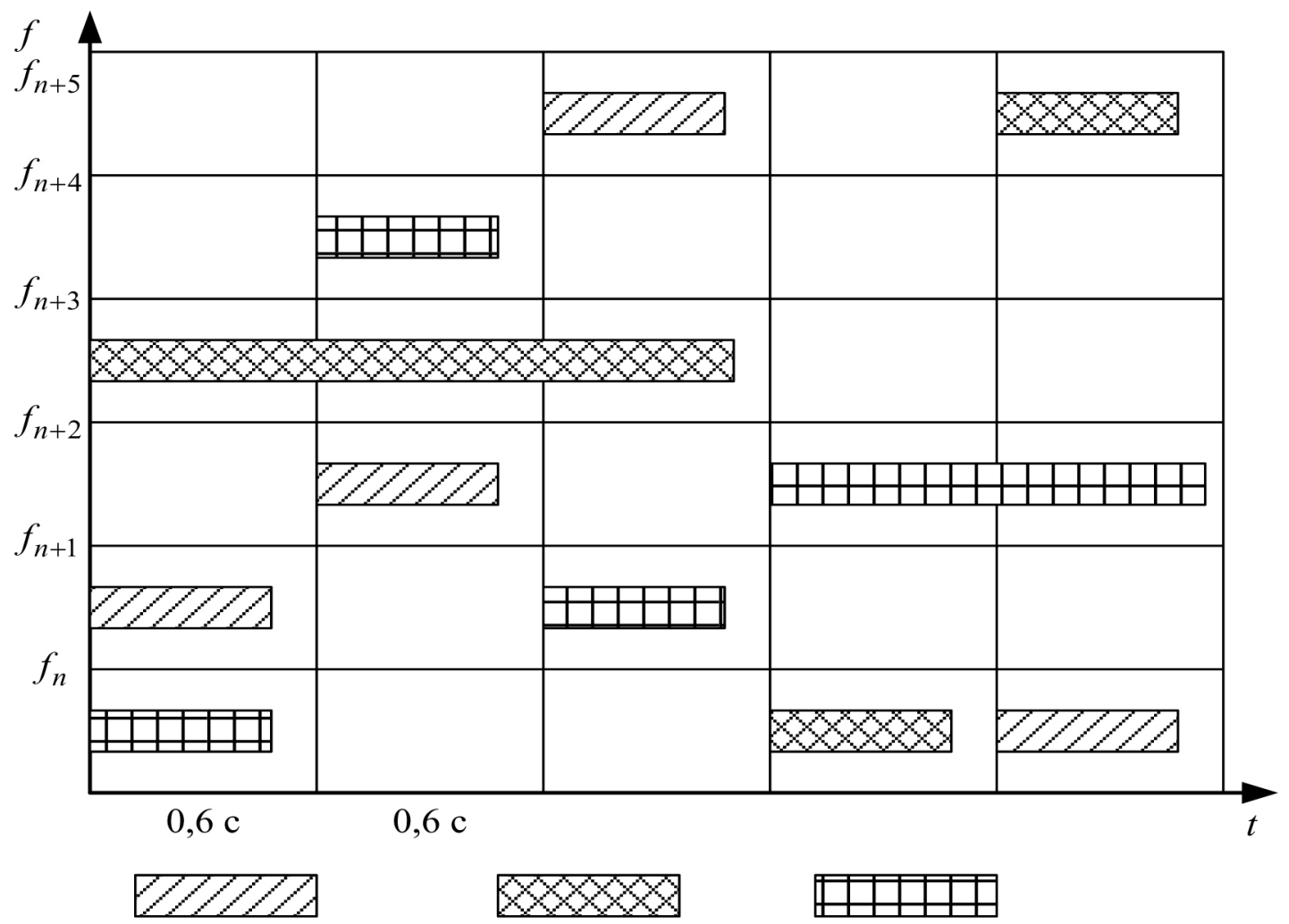

Fig. 1. Wireless frequency time diagram of module operation the electronic control system of aviation engine

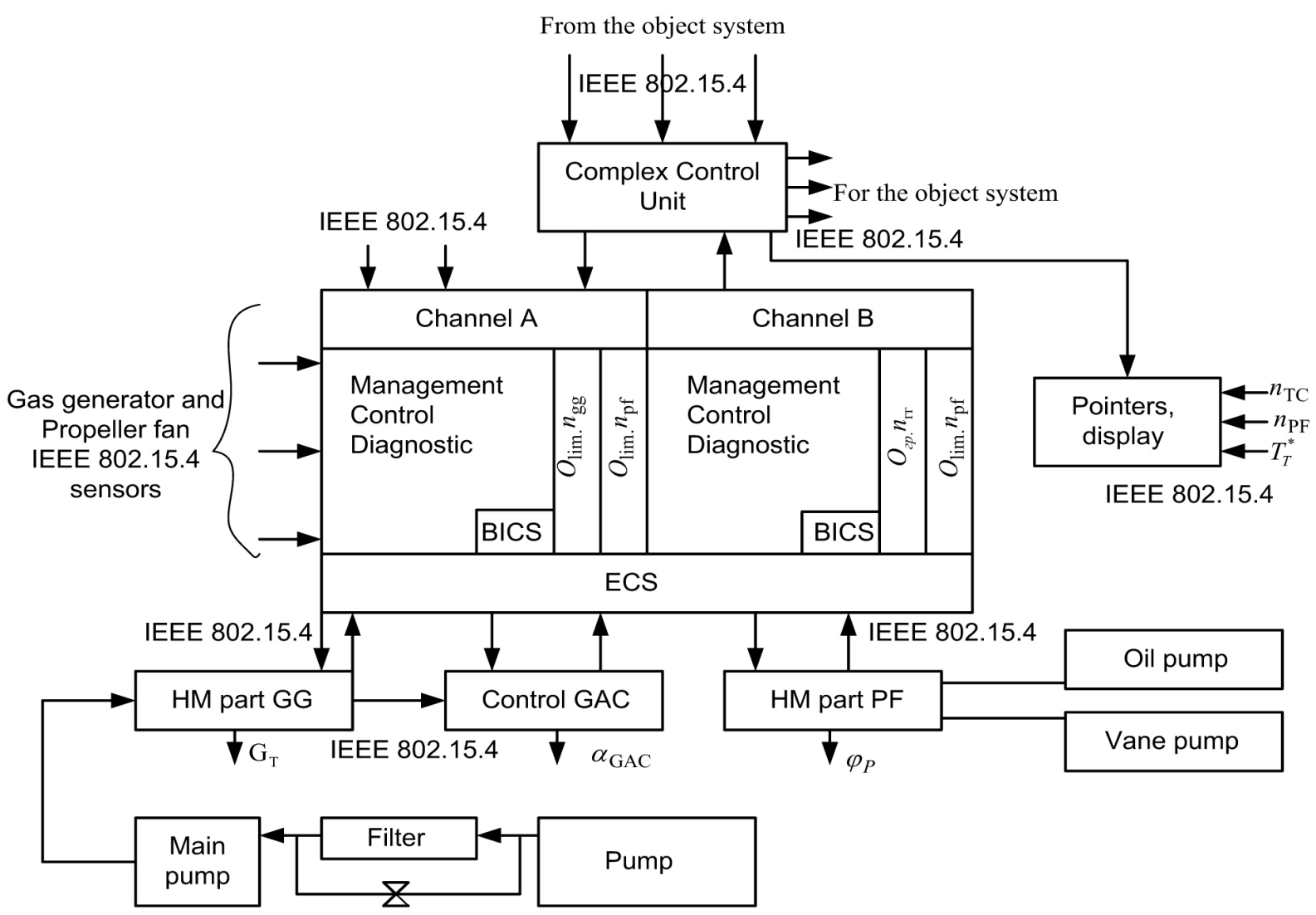

Fig. 2. Structure scheme ACS GTE 
master tells the driven, which of templates will be applied for data transmission.

Modern turbo propeller fan engine (TPFE) control systems are digital electronic, which perform all management functions by directly influencing the regulatory nodes of the engine [10]. Electronic controller performs the functions of control and control of gas generator and propeller fan.

In practice, different variants of designing an ACS are used:

- single-channel analog or digital systems with a developed hydromechanical part;

- two-channel digital system with simplified hydromechanical reserve;

- two-channel digital system without hydromechanical reserve.

Hydrochemical over-system protection can be used in the ACS (Fig. 2).

The system has two identical control channels $\mathrm{A}$ and $\mathrm{B}$, both of which performs the functions of automatic control of the gas generator (GG), propeller fan (PF) and control of the technical state of GH and HBV. Each channel has an autonomous protection system from the promotion of a free turbine $\left(O_{\text {lim }} n_{P F}\right)$,a gas generator $\left(O_{\lim } n_{G G}\right)$ and a built-in control system (BICS) that provides failures while reconfiguring the system and compensating failures.

The connection of the engine control system with the aircraft control systems is carried out through the airplane electronic control unit and the complexation with the help of digital communication lines.

In order to increase the reliability of the ACS sensors and electrohydraulic transducers are performed by two-channel (two-winding).

The fuel supply system includes a pumped centrifugal pump, a filter with a pass, a main gear unit or a plunger pump.

As a working fluid, the oil supplied by the pump located in the propeller regulator or the vane pump is used to control the blade rotation of the propeller. In the regulator pump, the oil is fed from the engine oil system, and the vane pump is fed from a separate oil tank.

\section{CONCLUSION}

The main approach for investigation the self-similarity of operating modes of the aviation engine and constructing the adaptive control systems with optimal algorithms the flexible scheme factorization traffic of wireless data transmission were considered.
In order to increase the efficiency of functioning ACS GTE, it is necessary to investigate the influence of multifractality on the quality parameters of a telecommunications network IEEE 802.15.4 traffic in mobile networks by solving the following main tasks:

- analysis the experimental IEEE 802.15.4 data and identification of mono and multifractal properties the traffic for protocols at various levels of the OSI model.

- estimation of the main parameters of the Legendre multifractal spectra of the investigated traffic including splitting functions, scaling index, and also scaling the parameters at various levels of aggregation traffic based on experimental data in order to develop analytical models of the multifractality of the investigated traffic.

- development of methods for analytic and numerical evaluation of the probability of exceeding the permissible time spent in the buffer as one of the main indicators of the quality of information transmission in telecommunications packet-switched networks and receiving the results that characterize the influence of the multifractal properties of IEEE 802.15.4 data on the characteristics of the quality of service of such communication networks.

Solving these tasks will allow achieving the main goal of the research: improving the quality of service the telecommunication networks with packet switching by accounting for the multifractal properties of telecommunications traffic during transmission IEEE 802.15.4 data in ACS aviation engines.

\section{REFERENCES}

1. Gurevich O.S. Automatic control systems of aviation gas turbine engine, Toru Press, 2010.

2. Securaplane Technology Inc. Wireless technology intra-aircraft wireless data bus for essential and critical applications. Access mode: https://www. securaplane.com/.

3. EUROCAE - standarts for future aviation. Access mode: https://www.eurocae.net.

4. Zhuravlev V.N., Papchenkov A.V., Borzov S.A. Methodical adequacy of the spectral model in the problem of technical diagnostics of rotor parts of GTE gearboxes. Bulletin of engine building, 2, 2014, 1-10. 
5. Tovkach S.S. Wireless technology for information exchange in systems adaptive control of aviation engines. Bulletin of engine building, 2, 2017, 24-28.

6. Chui K. Introduction to Wavelets, Mir, 2001.

7. Automated data processing. Access mode: http:// dic.academic.ru/dic.nsf/fin enc/19811.

8. Enchev S.V., Tovkach S.S. Method of multifractal formalism for analysis information in electonic control systems aircraft gas turbine engines. Proc. of the fifth world congress "Aviation in the XXI-st century", Ukraine, Kyiv, 2012, 1.5.12-1.5.15.

9. Daubechies I. Ten lectures on wavelets, Philadelphia, S.I.A.M., 1992.

10. Falaleev S.V. Modern problems of creating aircraft engines, 2012,106. 